\title{
Near-field light localization using subwavelength apertures incorporated with metamaterials
}

\author{
Damla Ates $*, 1$, Atilla Ozgur Cakmak ${ }^{1}$, Ekmel Ozbay \\ Nanotechnology Research Center, Department of Physics, Department of Electrical and Electronics Engineering, Bilkent University, 06800 Ankara, Turkey
}

\section{A R T I C L E I N F O}

Article history:

Received 1 November 2011

Accepted 28 February 2012

Available online 12 March 2012

\section{Keywords:}

Electromagnetic wave transmission

Diffraction, grating and apertures

Metamaterials

Resonators

\begin{abstract}
A B S T R A C T
We report strong near-field electromagnetic localization by using subwavelength apertures and metamaterials that operate at microwave frequencies. We designed split ring resonators with distinct configurations in order to obtain extraordinary transmission results. Furthermore, we analyzed the field localization and focusing characteristics of the transmitted evanescent waves. The employed metamaterial configurations yielded an improvement on the transmission efficiency on the order of $27 \mathrm{~dB}$ and $50 \mathrm{~dB}$ for the deep subwavelength apertures. The metamaterial loaded apertures are considered as a total system that offered spot size conversion ratios as high as 7.12 and 9.11 for the corresponding metamaterial configurations. The proposed system is shown to intensify the electric fields of the source located in the near-field. It also narrows down the electromagnetic waves such that a full width at half maximum value of $\lambda / 29$ is obtained.
\end{abstract}

(c) 2012 Elsevier B.V. All rights reserved.

\section{Introduction}

The transmission characteristics of light through apertures have been the subject of the studies for a long time. The earlier explanations based on the Kirchhoff scalar diffraction theory gave good results as long as the opening attained comparably larger values with respect to the operational wavelength, $\lambda$. In 1940s, $H$. Bethe addressed the shortcomings of the Kirchhoff scalar diffraction theory and developed a new approach that attempted to elucidate the electromagnetic wave transmission for the subwavelength apertures [1]. According to Bethe, the transmission efficiency scaled as $(r / \lambda)^{4}$ for an infinitesimally thin metallic screen with perfectly conducting walls extending to infinity, where $r$ stands for the radius of the subwavelength aperture. Both of the theories concentrated on the opaque screens that were constituted from the ideal material parameters and excluded the contributions of the evanescent surface waves on the transmission characteristics. It was only after the pioneering works at the turn of the century which pointed out the possibility of an extraordinary transmission through subwavelength aperture arrays that the attention of the scientific community has once again been directed towards the perforated metallic slits [2]. This era also marked the beginning of the scientific debates over the leading mechanisms of the extraordinary transmission. Initially, the extraordinary transmission was associated with the surfaceplasmon phenomenon at the dielectric-metal interface at optical wavelengths. Thereafter, several results targeting the extraordinary

\footnotetext{
* Corresponding author. Tel.: +90 5366526719.

E-mail address: damla@ee.bilkent.edu.tr (D. Ates).

1 The authors equally contributed.
}

transmission started to be reported at microwave frequencies as well as in optical wavelengths [3-7]. The observed surface waves at microwave frequencies are ascribed to the designer surface plasmons that exist due to the modification of the surface impedance by making use of the pure geometrical parameters [8]. Moreover, the researchers have investigated the effect of periodicity and size of the hole arrays. Scientists designed corrugations around the apertures and surfaces to establish an improved coupling $[9,10]$. The accumulated theoretical and experimental experiences enabled the extraordinary transmission to be attributed to the coupling of the leaky surface waves at both ends of the aperture through the evanescent waveguide modes of the subwavelength opening. The corrugated surfaces increased the transmission and provided better coupling conditions. Alternatively, Alu et al. suggested covering the aperture with a metamaterial slab in order to achieve superior coupling efficiencies [11]. On the other hand, Bilotti et al. proposed exploiting the resonance characteristics of the metamaterials, which in turn yielded the amplification of the electromagnetic fields around the aperture [12]. Since the metamaterials provide the field localization opportunity in the vicinity of the resonance frequency, they can be considered as a strong candidate for a prospective corrugation design that boosts the transmission efficiency. Accordingly, Aydin et al. experimentally showed a 740-fold transmission enhancement by inserting a split ring resonator inside the aperture [13]. Then, in one of our previous studies we discussed the physical origins of the enhancement in terms of the induced surface currents [14]. Recently, we have demonstrated a 70,000-fold enhancement experimentally and analyzed the parameters of the designed structure [15].

Independently, the localization theory has been developed in the 1960 s and light localization obtained in a wide range of areas from 
lasers to photonic crystals and gratings has gained a significant interest from the scientific community in the recent years [16,17]. One of the most striking ways to localize fields is using metamaterials. Nanowire metamaterial designs and asymmetrical split ring resonators were employed to localize the electric fields [18-20]. Cavities that resemble the counterparts in photonic crystal literature are utilized to confine the electromagnetic waves $[21,22]$. Conversely, there have been attempts to localize the fields outside the metamaterial specimen. This subject has been first attended by Pendry et al. under the topic of super lenses [23]. N. Fang and X. Zhang considered the imaging properties of the metamaterial super lenses and observed that the evanescent waves are strengthened at the exit side [24]. Furthermore, scientists such as Grbic et al. treated the negative index lens as a transmission line to realize the subdiffraction imaging [25]. Aydın et al. has achieved a strong localization by focusing the evanescent waves with the aid of negative index materials [26].
For the purposes of this paper we will be mainly concentrated on the field localization at the output side of a subwavelength hole and a subwavelength slit incorporated with two distinct metamaterial designs. The localized beam already results in extraordinary transmission. Firstly, the transmission mechanism offered by using two different metamaterial configurations will be discussed. Secondly, the confinement capabilities of the proposed structures will be investigated and compared.

\section{Split ring resonator configurations}

A single resonator embedded close to a subwavelength aperture is the key element in elevating the electromagnetic fields and will comprise the fundamental building block for the subwavelength field localization. The first configuration is illustrated in Fig. 1(a). The zoomed perspectives are also shown for convenience. The split ring
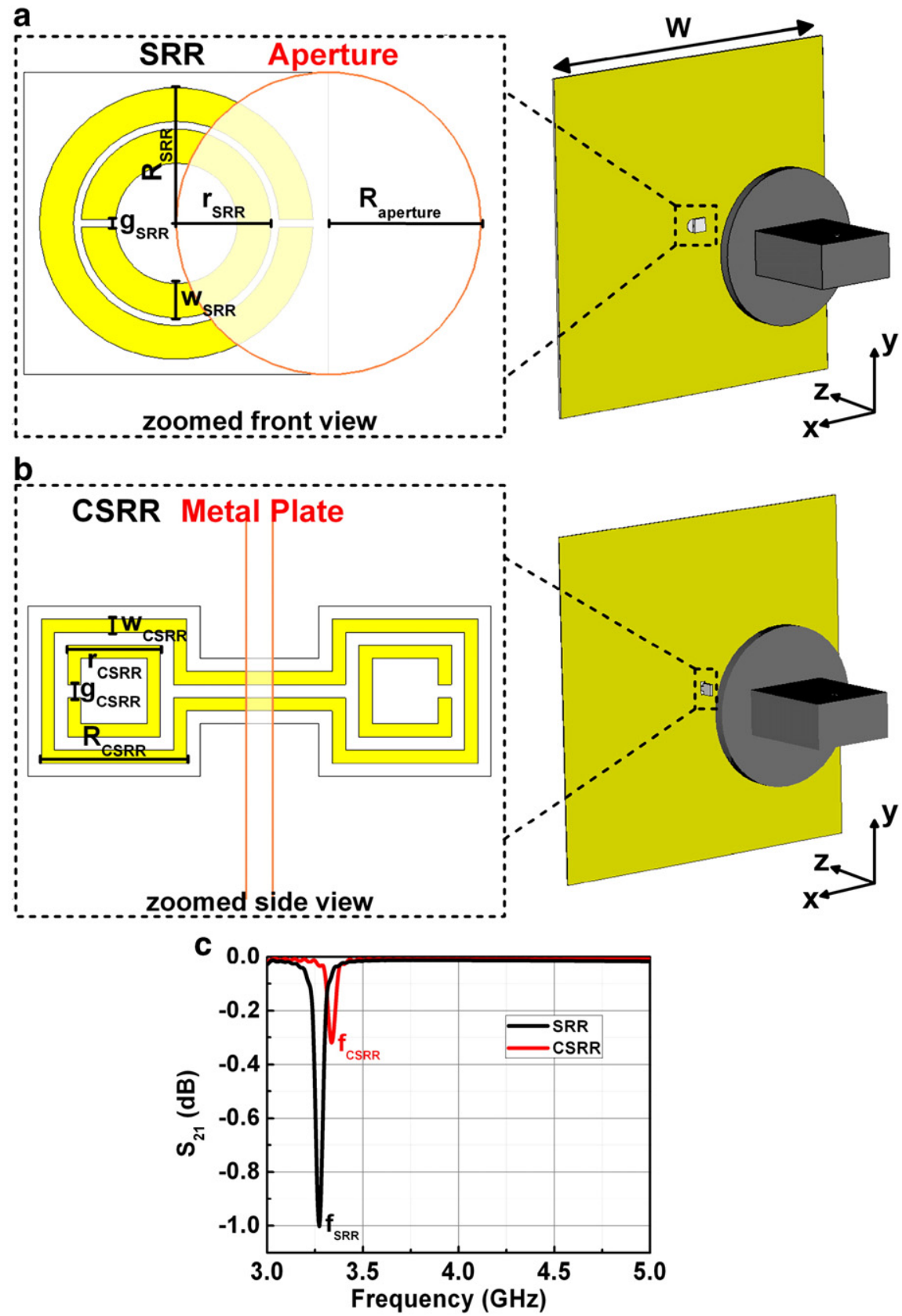

Fig. 1. (a) The SRR and the aperture, (b) the CSRR and the aperture, and (c) simulated transmission results ( $\mathrm{S}_{21}$ ) for the SRR (black line) and the CSRR (red line). 
resonator (SRR) is carefully aligned at the midpoint of the aperture such that the gaps of the SRR, $g_{S R R}$ are parallel to $y$-axis. The propagation direction is $z$-axis and the aperture is illuminated with a waveguide in the experiments. This particular waveguide is modeled in CST Microwave Studio and shown in gray color. The aperture has a radius $R_{\text {aperture }}=4 \mathrm{~mm}$ and resides on a square plate with a width $\mathrm{W}=200 \mathrm{~mm}$. The SRR gaps are $\mathrm{g}_{\mathrm{SRR}}=0.2 \mathrm{~mm}$, while the inner and outer radius of the structure are $\mathrm{r}_{\mathrm{SRR}}=2.7 \mathrm{~mm}$ and $\mathrm{R}_{\mathrm{SRR}}=3.6 \mathrm{~mm}$. The metal width is $\mathrm{w}_{\mathrm{SRR}}=0.9 \mathrm{~mm}$.

Subsequently, we have designed split ring resonators that have connecting bars and the configuration is depicted in Fig. 1(b). These connected SRRs are labeled as CSRR. The samples are fabricated in a similar way to the previous design. They are deposited on a dielectric printed circuit board (PCB) with a $1.6 \mathrm{~mm}$ thickness. The connected bar length is $l_{\mathrm{CSRR}}=5.5 \mathrm{~mm}$. The remaining parameters for the individual SRRs are $\mathrm{g}_{\mathrm{CSRR}}=\mathrm{w}_{\mathrm{CSRR}}=0.5 \mathrm{~mm}$, and $\mathrm{r}_{\mathrm{CSRR}}=3.5 \mathrm{~mm}$, RCSRR $=5.5 \mathrm{~mm}$. The area of the rectangular aperture is $3 \times 7.5 \mathrm{~mm}^{2}$. The CSRR is inserted midway into the aperture. The thicknesses of the deposited copper are the same in both configurations and it is equal to $30 \mu \mathrm{m}$.

The resonance frequencies of the regarding resonators are shown in Fig. 1(c). The launched beam has an electric field component parallel to $y$-axis. Then, the gap of the SRR captures the incoming electric field which induces circulating currents and creates accumulated charges at the resonance condition. A similar action will take place for CSRR which will only result in smaller resonance strength in contrast to the single SRR. The effect of shortening the SRRs destroys the charge accumulation at the resonance frequency. As it can be seen from Fig. 1(c), the shortened SRRs in CSRR do not totally lose their resonance owing to the fact that shortening is performed over a longer path. Nevertheless, a significant portion of the charges will leak away through the connection bars. One might also wonder the overall shallow depths of the transmission results. The attained $S_{21}$ results are one order of magnitude higher (in $\mathrm{dB}$ scale) than the usually inspected values for conventional SRRs around the resonance frequency. Given that a single resonator will be positioned around the aperture in the proceeding sections, the transmission characteristics have been investigated for a single SRR in order to avoid the contributions coming from the coupling mechanisms in a SRR array. Therefore, the SRR and CSRR are illuminated with a beam of finite width that can circumvent the resonators and reach the detector. Periodic boundary conditions are not allowed in the simulations. Consequently, the resonators could capture a limited amount of the propagating waves. Yet, we obtain the individual resonance frequencies of our configurations as $\mathrm{f}_{\mathrm{SRR}}=3.27 \mathrm{GHz}$ and $\mathrm{f}_{\mathrm{CSRR}}=3.33 \mathrm{GHz}$.

\section{Transmission enhancement phenomenon}

Once the resonator is placed in the vicinity of the subwavelength aperture simulated and measured transmission characteristics are plotted in Fig. 2(a) and (b) for the SRR configuration, respectively. The transmission enhancement frequency turned out to be $3.6 \mathrm{GHz}$ in the simulation results, whereas a peak is spotted at $3.56 \mathrm{GHz}$ in the experiments. There is a good agreement between the experimental and simulation based results in terms of the general transmission characteristics. This subwavelength aperture has a cutoff frequency for the lowest allowed modes at almost $22 \mathrm{GHz}$. Then, the aperture can be visualized as a LC tank with a dominant inductive character which forces the opening to act as a high pass filter [27]. According to Bethe, we would anticipate a dramatically weak transmission at the output of the subwavelength aperture in the absence of the resonator. Instead of the electrically large cover suggested by Alu et al. in Ref. [11], we make use of the resonance mechanism of a single negative material as pointed out in Ref. [12]. It was indicated in Ref. [12] that only the effective permeability would be the determining constitutive parameter and a transmission enhancement could also be sustained at another frequency where the reduced permeability $\mu=-1$ is feasible. However, the detected transmission enhancement is solely
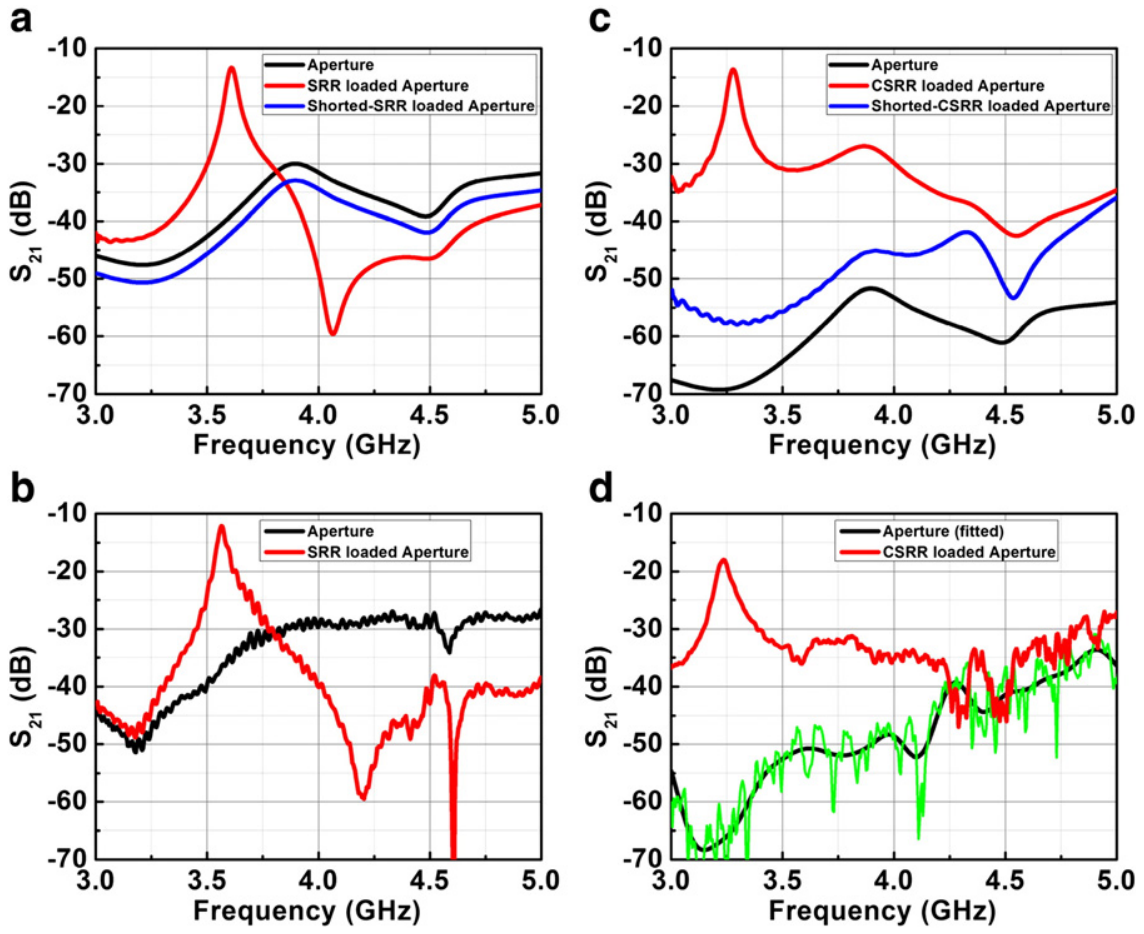

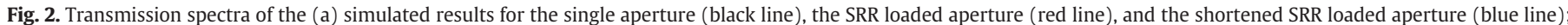

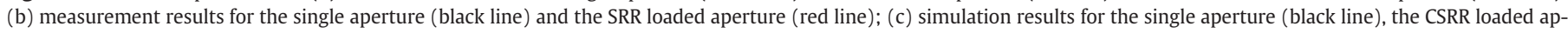

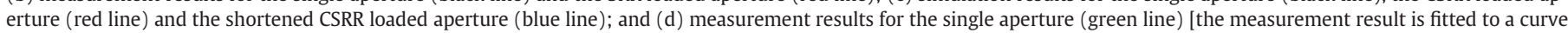
(black line) because of the sudden ripples] and the CSRR loaded aperture (red line). 
due to the induced resonance of the SRR for our configuration which offers approximately a $27 \mathrm{~dB}$ improvement in the transmission figures on top of the subwavelength aperture. A parallel magnetic dipole and a perpendicular electric dipole (with respect to the screen) are the responsible dipole moments for the transmission through the subwavelength opening. In our current configuration, the strong dipole moments are along $y$-axis for the electric and $z$-axis for the magnetic counterpart. Thus, we do not have a chance to elevate drastically the amplitudes of the original dipole moments of the subwavelength aperture around the resonance frequency. Nonetheless, the induced dipoles in the SRR cancel out the equivalent dipole of the subwavelength aperture in the neighborhood of the transmission enhancement frequency owing to the bianisotropy of the SRR, as indicated in Ref. [28]. Thus, we encounter a dip in the transmission graph inside the band starting from $4 \mathrm{GHz}$ up to $4.5 \mathrm{GHz}$. Finally, the shortened SRR loses its magnetic resonance and causes an augmentation in the loss figures, which further verifies the resonance based explanations.

In the meantime, the transmission characteristics have been laid out for the CSRRs in Fig. 2(c) and (d). The cutoff wavelength of the slit is at even higher frequencies $(42.8 \mathrm{GHz})$. As a result, smaller amount of transmission is detected for the subwavelength aperture case. The experiments are carried out with horn antennas connected to HP 8510C Network Analyzer. Absorbers have been placed in order to minimize the diffractions at the edges of the metallic screen, which can easily outbalance the main signal. Such effects trigger the ripples in the measured results in Fig. 2(d). Similar scenarios had been investigated in Ref. [15]. In contrast to the real life problem, we can numerically tackle this particular difficulty with perfectly absorbing boundaries. The resonance mechanism presents a transmission enhancement higher than $50 \mathrm{~dB}$ at the enhancement frequency of $3.28 \mathrm{GHz}$. Now, the transmission mechanism depends on the connected bars and we do not detect a transmission dip just after the enhancement frequency. The transmission figures stay improved over $20 \mathrm{~dB}$ with the addition of the CSRR on top of the single slit transmission. Lastly, the CSRR had been shortened from the midpoint of the connecting bars. In the end, this shortening of the connecting bars proved to have a comparable influence on the transmission spectra by destroying the magnetic resonance of the individual SRRs.

Fig. 3 is presented in order to shed light to the underlying physical mechanisms of the transmission enhancement phenomenon. The induced surface currents in the vicinity of the aperture are illustrated at the resonance frequency in Fig. 3(a). Then, the addition of the SRR strengthens an electric dipole along the $y$-axis. However, this induced dipole and thereby the induced surface currents are nullified at a certain degree by the image current formation on the metallic screen, as shown in Fig. 3(a). Consequently, the shaded area will be the main contributor of the induced currents whose function will be to transmit the impinging field to the exit side. In other words, the shaded area will play the role of an effective aperture for an ordinary antenna. In a very general manner the effective aperture of an antenna is defined as $A_{\text {eff }}=P_{\text {out }} / P_{\text {inc }}=G \lambda^{2} / 4 \pi$, where $P_{\text {out }}$ is the delivered power by the antenna, $P_{\text {inc }}$ is the incident power in terms of watts per unit area and $G$ is the antenna power gain. The effective aperture is proportional to the operational wavelength. The SRR together with the aperture constitutes a new transmission system. This is the main reason behind the mismatch between the given resonance frequency of the isolated SRR (see Fig. 1(c)) and the transmission enhancement frequency of the total system. Such a shift has also been reported in Ref. [12]. Unlike the cases in the earlier studies [12-14] the SRR's resonance is not magnetically excited and the SRR is directly facing the metal screen. These factors found the ground for a more pronounced shift to be observed between the self-resonance frequency and the transmission enhancement frequency. Then, an alteration of the exact location of the SRR directly determines the enhancement frequency. We have not observed a strong difference between the experimental and numerical values but a small misalignment would

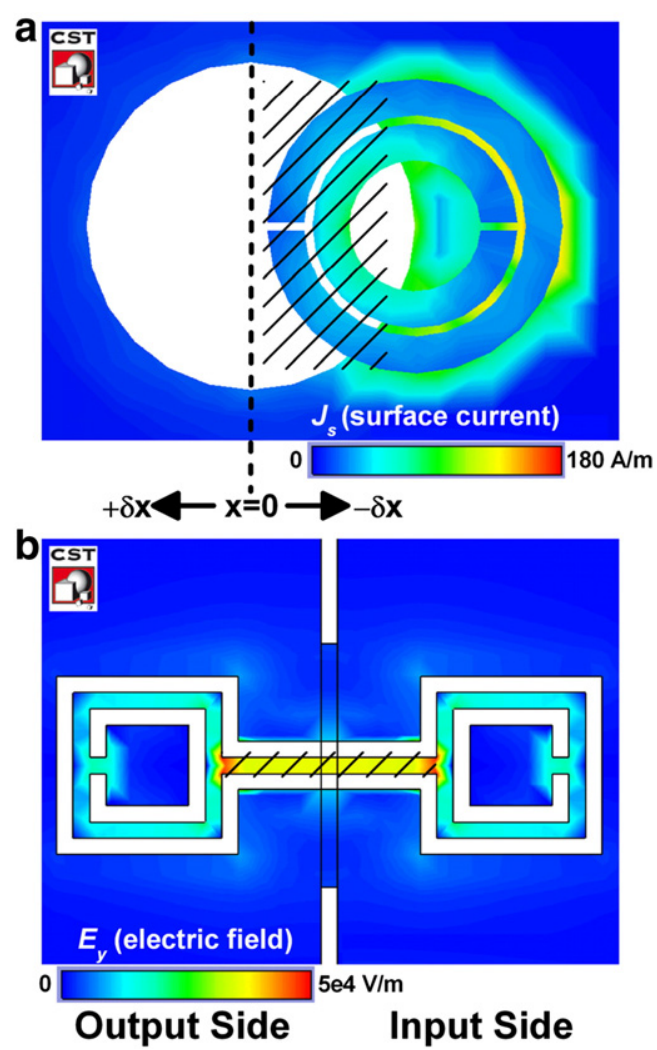

Fig. 3. (a) Induced surface currents on the SRR at $f=3.6 \mathrm{GHz}$. The shaded region encompasses the effective aperture of the overall SRR subwavelength aperture system (media 1). (b) The electric fields $\left(E_{y}\right)$ in the vicinity of the subwavelength aperture at $f=3.28 \mathrm{GHz}$. The shaded region emphasizes the strong spatial confinement of the electric fields inside the aperture (media 2 ).

be likely to produce quite different transmission characteristics. Ref. [14] considered the misalignments only in one dimension and showed that a shift of $\delta x_{1}=-0.1 \mathrm{~mm}$ and $\delta x_{2}=+0.3 \mathrm{~mm}$ would move the transmission enhancement frequency to the higher and lower frequencies from its original location respectively, as predicted from the qualitative effective aperture discussions. The transmission enhancement values attained lower figures for those cases, since the maximum electric dipole can be induced when the SRR is carefully positioned at the midpoint of the aperture assuming that the aperture is perfectly aligned with the incoming illumination. The other way of modifying the effective aperture size of the system is to tune the radius of the opening. It was also depicted in Ref. [14] that the transmission enhancement frequencies spanned a band from $3.3 \mathrm{GHz}$ to $6 \mathrm{GHz}$ for the corresponding aperture radii ranging from $2.8 \mathrm{~mm}$ up to $7 \mathrm{~mm}$.

Fig. 3(b) shows the calculated electric fields around the aperture at the designated resonance frequency. The CSRR can be regarded as a loop antenna connecting the two sides of the aperture. The connecting bars conduct the induced currents over a broad range of frequency. The induced currents continue to be shared outside the enhancement band. We are comparably much less limited by the resonance bandwidth of the structure. An electric dipole parallel to the screen is again induced. This time different from the SRR case, a magnetic dipole parallel to the screen is also excited and the interactions with the screen are comparably reduced. In turn, the transmission enhancement frequency shifted relatively less from the isolated resonator configuration in contrast to the SRR case. We do not have a strong dependency to the geometrical sizes of the aperture. The incoming fields are captured by the loop antenna and the fields are safely guided to the output plane. The shaded region localizes the fields during the transmission of the electromagnetic fields. Eventually, the 
connecting bars play the role of a subwavelength waveguide. The width of this particular waveguide turns out to be as small as $\lambda / 180$ at the transmission enhancement frequency. The fields are confined to this deep subwavelength waveguide for a propagation length of $\lambda / 18$. As it is previously discussed in Ref. [15], the inner loops of the individual SRRs do not play a major part in the transmission enhancement. The magnetic dipole is permanently obstructed when the outer loops are shortened and the waveguiding effect of the highly confined electric fields is thereby demolished. At these regarding peaks not only we obtain a high transmission but we also have a chance to localize the evanescent wave at the exit side of the aperture. In the next section, the discussions will be devoted to the examination of these scenarios.

\section{Field distributions and highly localized fields at the output plane}

In this part, we analyzed the characteristics of the near field localization around the resonance frequencies. The fields at the output side have been numerically collected and presented in Figs. 4 and 5 simultaneously. Fig. 4 entails the SRR loaded subwavelength hole while Fig. 5 involves the CSRR incorporated subwavelength slit. Figs. 4(a) and 5(a) exhibit the field distribution on the $x-z$ planes, whereas Figs. 4(b) and 5(b) portray the field distribution on the $y-z$ planes at their respective resonance frequencies in $\mathrm{dB}$ scale. All of the field distributions are normalized with respect to the maximum attained value in the concerning scenarios. The cutoff frequencies of the subwavelength hole and slit were calculated to be well below the operational frequency range. The transmitted fields in Figs. 4 and 5 are originally the evanescent modes of the subwavelength openings. Therefore, the fields decay dramatically in the direction of propagation. Consequently, intensified fields are localized in the near field zone of the aperture. Yet, the overall configurations propose to restrict the transmitted evanescent modes only on the $x-z$ planes. For instance, the loop antenna nature of the CSRR compels the fields to radiate isotropically on the $y-z$ planes, while the radiation is not allowed along the induced magnetic dipole direction ( $x$-axis), which is clearly observed in Fig. 5(a) [29]. Accordingly, the field profiles are plotted in Figs. 4(c) and 5(c) in order to explore the localization capabilities of the total systems. The minimum realizable full width at half maximum (FWHM) values of the overall systems are revealed as $\lambda / 23$ and $\lambda / 29$ for the SRR and CSRR incorporated approaches, respectively. The excitation source with FWHM values around $\lambda / 3$ is down converted by taking advantage of the subwavelength resonators placed in the reactive near field zone of the illumination. Then, the precise beam spot-size conversion values are calculated to be 7.12 and 9.11 for the systems with SRR and CSRR, correspondingly. It might not sound interesting to come across such a focusing effect at the output side of the subwavelength apertures. Nevertheless, the proposed designs have not only amplified the evanescent mode, but also narrowed down the outgoing fields exceeding the capabilities of the subwavelength openings, as shown in Figs. 4(c) and 5(c). We numerically integrated the intensity profiles on $x-y$ planes along the direction of propagation (z-axis). Figs. 4(d) and 5(d) explicitly demonstrate that the power values drop rapidly in the direction of propagation. Approximately $10 \%$ of the initial power is conserved in the case of SRR loaded apertures while around 20\% is preserved for the configuration with the CSRR. On the other hand, when the FWHM values are inspected, $\lambda / 5$ FWHM is still feasible at a distance of $0.15 \lambda$ away from the aperture loaded with the SRR. The same FWHM values can be observed in the CSRR at a location $0.1 \lambda$ away from the metallic screen. The SRR configuration extracts more intensity from the subwavelength structure by focusing down the amplified evanescent fields, which can even get higher values than the
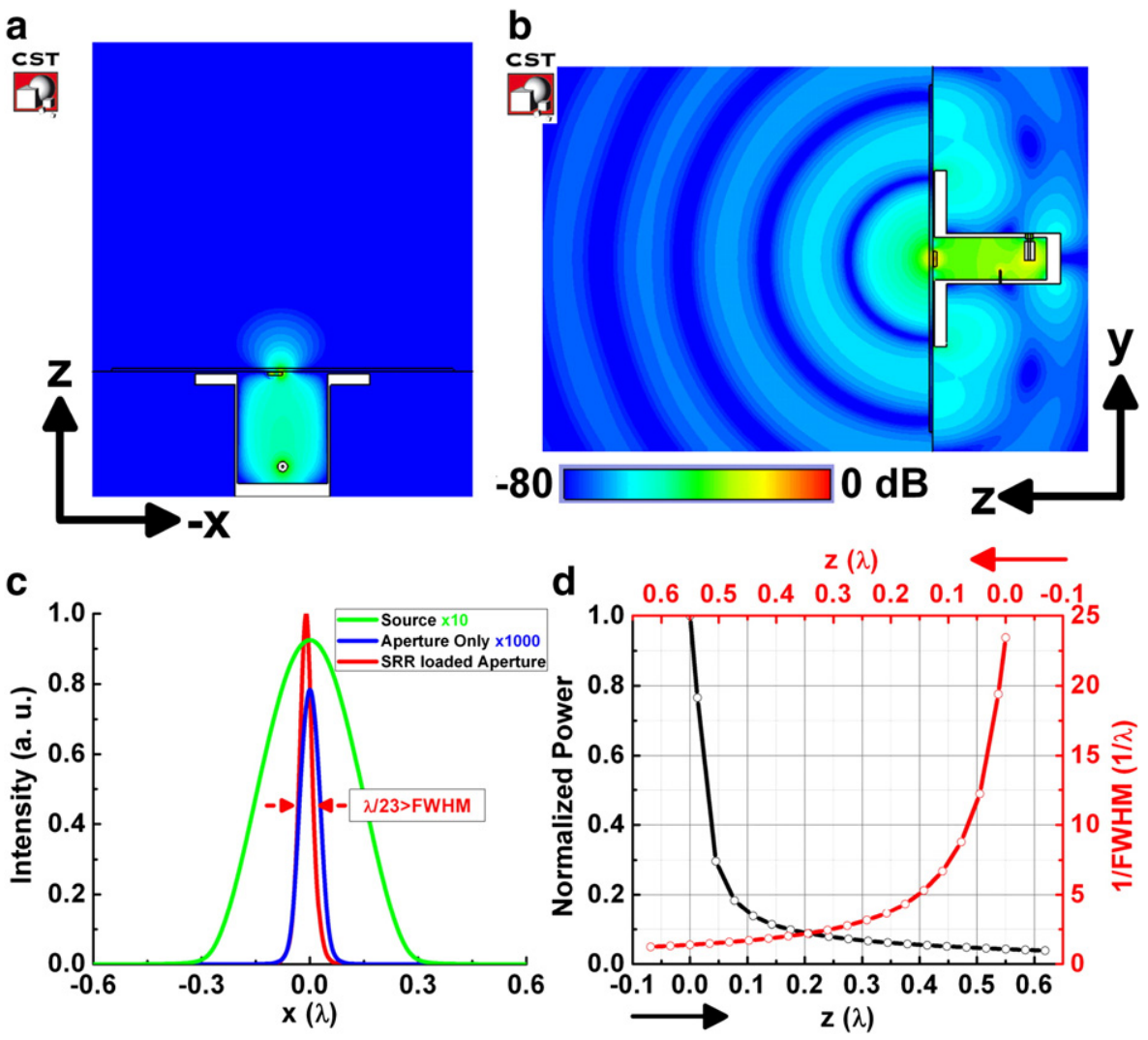

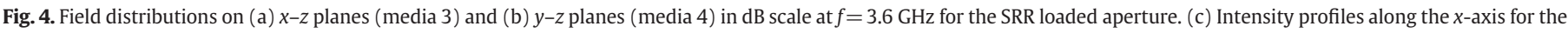

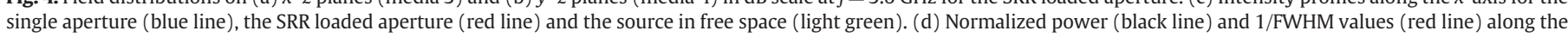
propogation direction ( $z$-axis). 
a

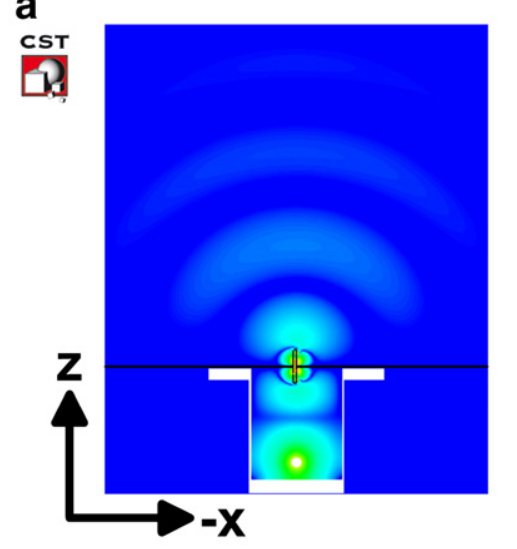

C

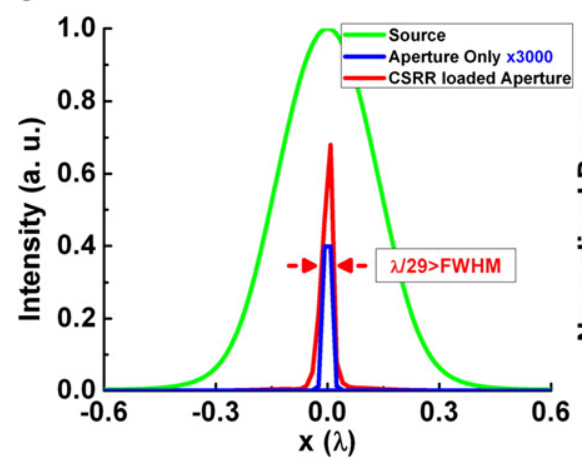

b

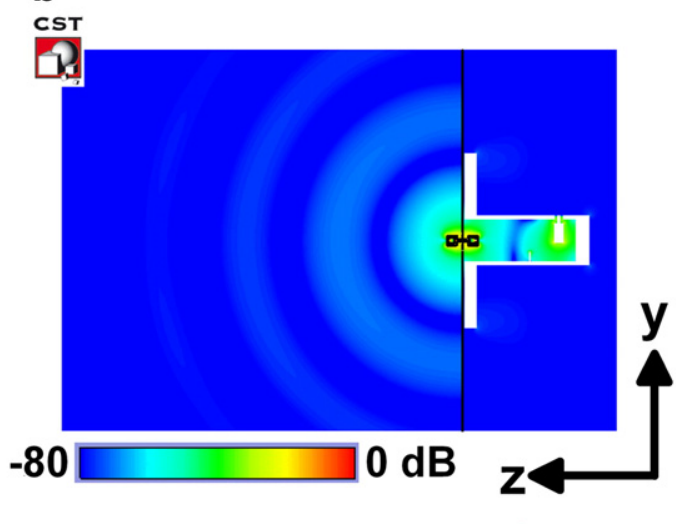

d $\quad \mathrm{z}(\lambda)$

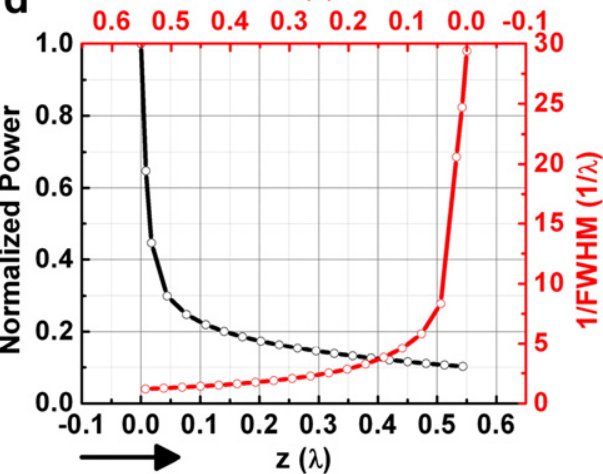

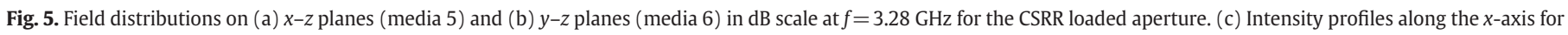

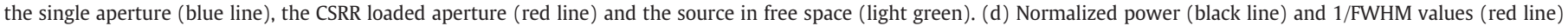
along the propogation direction ( $z$-axis).

source itself, as it can be seen in the field profiles of Fig. 4(c). Conversely, the CSRR structure promises an improved spot size conversion ratio when the FWHM values are taken into consideration.

\section{Conclusions}

Transmission enhancement through subwavelength apertures is still a challenging topic in experimental physics. In this paper, we have demonstrated alternative methods for improving the transmission figures of the otherwise opaque screens. The resonance nature of the SRR and CSRR is exploited in order to boost the transmission efficiency of the evanescent modes by factors of $27 \mathrm{~dB}$ and $50 \mathrm{~dB}$, respectively. The physical origins of the extraordinary transmission are discussed in details. It is emphasized that the SRR loaded holes must be regarded as a combined system which has a certain effective aperture size. Through this effective aperture the leaky modes are transmitted to the outer plane. On the other hand, the CSRR loosens the dependency on the geometrical parameters of the aperture while behaving like a loop antenna. The captured fields are guided via the connecting bars to the exit side. Furthermore, we investigated the field distributions of the outer plane. The overall system offers spot conversion ratios as high as 7.12 and 9.11 for the SRR and CSRR designs, respectively. FWHM values down to $\lambda / 29$ are demonstrated numerically in the near field of the excitation source by attaching the CSRR to the subwavelength slit.

The proposed designs are simulated and measured at microwave frequencies while bearing in mind the prospective design adaptable for optical wavelengths. An analogy which will be suitable for the optical regime would prove to find essential application fields ranging from sensors, biophotonics, SNOM and subwavelength imaging. The horn antennas have to be replaced with the optical light sources such as lasers. The designer will encounter the additional losses coming from the material properties of the metals at optical wavelengths and will have to overcome the difficulties of the nanofabrication. Nevertheless, a careful design is expected to bring numerous novelties on top of the conventional systems for those who are interested in the near-field optics.

\section{Acknowledgment}

This work is supported by the projects DPT-HAMIT, EU-PHOME, EU-N4E, NATO-SET-181 and TUBITAK under project nos., 107A004, 107A012 and 109E301. One of the authors (E.O.) also acknowledges partial support from the Turkish Academy of Sciences.

\section{References}

[1] H.A. Bethe, Physical Review 66 (1944) 163.

[2] T.W. Ebbesen, H.J. Lezec, H.F. Ghaemi, T. Thio, P.A. Wolff, Nature 391 (1998) 667.

[3] L. Martin-Moreno, F.J. Garcia-Vidal, H.J. Lezec, K.M. Pellerin, T. Thio, J.B. Pendry, T.W. Ebbesen, Physical Review Letters 86 (2001) 1114.

[4] J.A. Porto, F.J. Garcia-Vidal, J.B. Pendry, Physical Review Letters 83 (1999) 2845

[5] K.G. Lee, Q.-H. Park, Physical Review Letters 95 (2005) 103902.

[6] M. Beruete, M. Sorolla, I. Campillo, J.S. Dolado, L. Martin-Moreno, J. Bravo-Abad, F.J. Garcia-Vidal, IEEE Transactions on Antennas and Propagation 53 (6) (2005) 1897.

[7] F.J. Garcia-Vidal, H.J. Lezec, T.W. Ebbesen, L. Martin-Moreno, Physical Review Letters 90 (2003) 213901.

[8] J.B. Pendry, L. Martin-Moreno, F.J. Garcia-Vidal, Science 305 (2004) 847.

[9] A. Degiron, T.W. Ebbesen, Optics Express 12 (2004) 3694.

[10] E. Ozbay, Science 311 (5758) (2006) 183.

[11] A. Alu, F. Bilotti, N. Engheta, L. Vegni, IEEE Transactions on Antennas and Propagation 54 (2006) 1632.

[12] F. Bilotti, L. Scorrano, E. Ozbay, L. Vegni, Journal of Optics A: Pure and Applied Optics 11 (2009) 114029.

[13] K. Aydin, A.O. Cakmak, L. Sahin, Z. Li, F. Bilotti, L. Vegni, E. Ozbay, Physical Review Letters 102 (2009) 013904.

[14] A.O. Cakmak, K. Aydin, E. Colak, Z. Li, F. Bilotti, L. Vegni, E. Ozbay, Applied Physics Letters 95 (2009) 052103. 
[15] D. Ates, A.O. Cakmak, E. Colak, R. Zhao, C.M. Soukoulis, E. Ozbay, Optics Express 18 (2010) 3952.

[16] X. Jiang, C.M. Soukoulis, Physical Review Letters 85 (2000) 70.

[17] X. Jiang, C.M. Soukoulis, Physical Review E 65 (2002) 025601.

[18] T.S. Kao, F.M. Huang, Y. Chen, E.T.F. Rogers, N.I. Zheludev, Applied Physics Letters 96 (2010) 041103.

[19] S. Savo, N. Papasimakis, N.I. Zheludev, Quant. Elect. and Laser Sci. Conference, OSA, 2011.

[20] T.S. Kao, S.D. Jenkins, J. Ruostekoski, N.I. Zheludev, Physical Review Letters 106 (2011) 085501.

[21] H. Caglayan, I. Bulu, M. Loncar, E. Ozbay, Optics Letters 34 (2009) 88.
[22] M. Bayindir, B. Temelkuran, E. Ozbay, Physical Review Letters 84 (2000) 2140

[23] J.B. Pendry, Physical Review Letters 85 (2000) 3966.

[24] N. Fang, X. Zhang, Applied Physics Letters 82 (2003) 161.

[25] A. Grbic, G.V. Eleftheriades, Physical Review Letters 92 (2004) 117403.

[26] K. Aydin, I. Bulu, E. Ozbay, Optics Express 13 (2005) 8753.

[27] S. Ramo, J.R. Whinnery, T. Van Duzer, Fields and Waves in Communication Electronics, John Wiley \& Sons, Inc, 1994.

[28] R. Marques, F. Mesa, J. Martel, F. Medina, IEEE Transactions on Antennas and Propagation 51 (10) (2003) 2572.

[29] C.A. Balanis, Antenna Theory Analysis and Design, John Wiley \& Sons, Inc, 2005. 